\title{
Nephelauxetic Effect Revisited
}

\author{
ANDREI L. TCHOUGRÉEFF, ${ }^{1,2}$ RICHARD DRONSKOWSKI ${ }^{2}$ \\ ${ }^{1}$ Poncelet Laboratory, Independent University of Moscow, Moscow Center for Continous \\ Mathematical Education, Moscow 119991, Russia \\ ${ }^{2}$ Institute of Inorganic Chemistry, RWTH Aachen, Aachen D-52056, Germany
}

Received 1 October 2008; accepted 3 November 2008

Published online 10 April 2009 in Wiley InterScience (www.interscience.wiley.com).

DOI 10.1002/qua.21989

\begin{abstract}
We readdress the well-known nephelauxetic effect in coordination compounds of transition metal ions and analyze its possible origins presented in the literature. The initial hypothesis was to ascribe the reduction of the effective Racah parameters $B$ and $C$ of the electron-electron interaction in the complexes as compared to their respective free ion values (which is the essence of the nephelauxetic effect) to the expansion of $d$-orbitals in the complex because of their quantum mechanical mixing with the orbitals of the ligands leading to delocalization. This picture necessarily leads to a rigid positive correlation between the amount of the $d$-shell splitting controlled by the same delocalization and the amount of the renormalization of the interaction parameters. In fact, such a rigid relation does not exist and the so-called spectrochemical and nephelauxetic series of the ligands composed according to the aforementioned amounts do not coincide in many points. An alternative explanation based not on the delocalization, but on polarization of the ligands had been proposed at the same time as the delocalization based one. Realistic estimates had been obtained on this basis, but the scheme had never been implemented on the atomic scale, which is necessary to enable renormalization of the aforementioned interaction parameters as a "built-in" function of quantum chemical software. The required atomic resolution formulation of the polarization-based model of the nephelauxetic effect is constructed in this work, in which a relation of such an approach to the general problematics of the "next generation" of semiempirical methods of quantum chemistry is also discussed. (ㅇ 2009 Wiley Periodicals, Inc. Int J Quantum Chem 109: 2606-2621, 2009
\end{abstract}

Key words: effective Hamiltonian crystal filed theory; nephelauxetic effect; next generation of semiempirical methods; transition metal complexes

\footnotetext{
Correspondence to: A. Tchougréeff; e-mail: tch@elch.chem.msu.ru

Contract grant sponsor: RFBR.

Contract grant number: 07-03-01128.

Contract grant sponsor: DFG.

Contract grant number: DR 342/20-1.
} 


\section{Introduction}

A mong the words to be learned by heart by first-year inorganic chemistry students fascinated by the bright colors of the coordination compounds of transition elements, the term "nephelauxetic" (effect) is one of the most intriguing, but unfortunately being rarely readdressed during further years of studies or even professional carriers. This beautiful Greek word introduced into theoretical inorganic chemistry by Jaurgensen [1] following the advice of Kaj Barr-the prominent Danish orientalist-refers to the mental picture according to which the cloud $(\nu \varepsilon \varphi \varepsilon \varepsilon \lambda \eta)$ of $d$-electrons expands $(\alpha v \xi \varepsilon \iota \nu)$ when a transition metal ion (TMI) becomes a central one in a coordination compound, where it is surrounded by various other inorganic or organic ions or molecules, rather a free one. The consequences of the coordination process for the $d$-shell are in principle twofold: before the already mentioned expansion of the $d$-orbitals the splitting of the $d$-levels degenerate in the free ions takes place, being much more important both qualitatively and energetically, namely, the splitting is ultimately responsible for the mentioned colors of the transition metal complexes (TMCs). Its qualitative picture is explained by the crystal filed theory (CFT) originally proposed by Bethe [2] and numerously represented in various other sources adjusted for the chemical problem setting [3-6] (originally CFT had been developed for the transition metal impurity ions in the crystals). The CFT bases on the very natural picture of what happens in TMCs-all interesting events (low- energy excitations) - are localized in the $d$-shell of a central TMI, whereas other atoms or groups provide some external, originally purely electrostatic, field responsible for the splitting. In such a formulation of the CFT known as its ionic model, and treating the splitting of the $d$-shell as a pure electrostatic effect, the CFT faces a serious problem: the splitting parameters cannot be correctly estimated. Although the symmetry is perfectly reproduced even by this simplistic scheme, it turns out that quantitatively the ionic model gives at best $20 \%$ of the observed splitting even if unrealistically large effective charges are ascribed to the ligands. This happens due to the oversimplified description of the TMI's environment (ligands). It is not thus surprising that the heaviest strike upon the CFT from the (semi)quantitative side was given by TMC spectroscopy in 30s of the last century. Spectroscopic experiments allowed to range different ligands according to the strengths of the crystal fields induced by them (the $10 D q$ parameter) to the so-called spectrochemical series [3-6] having (with many omissions) the following form:

$$
\begin{aligned}
\mathrm{I}^{-}<\mathrm{Br}^{-}<\mathrm{S}^{2-}<\mathrm{N}^{3-}<\mathrm{F}^{-} & <\mathrm{OH}^{-}<\mathrm{Cl}^{-} \\
< & \frac{1}{2} \mathrm{Ox}^{2-}<\mathrm{O}^{2-}<(1) \\
<\mathrm{H}_{2} \mathrm{O}<\mathrm{SCN}^{-}<\mathrm{NH}_{3} \text { py } & <\frac{1}{2} \mathrm{En} \\
<\mathrm{SO}_{3}^{2-} & <\mathrm{NO}_{2}^{-}<\mathrm{CN}^{-}<\mathrm{CO} .
\end{aligned}
$$

From this one can see that the crystal fields splittings are systematically weaker for charged ligands than for the uncharged ones with the utter example of $\mathrm{CO}$ inducing the strongest crystal field, but bearing neither charge nor even noticeable dipole moment. Thus, the relative strengths of the crystal fields observed in the experiment cannot be explained by the ionic model of the environment. These observations clearly indicate that purely electrostatic effects may be only of minor significance in determining the strength of the effective crystal field felt by the $d$-shell.

The unsatisfactory situation with the CFT estimates called for the development of the ligand field theory (LFT) [3, 4], trying to include the ligands on a more realistic basis. In its simplest version, it assumes that it is enough to consider the valence shell of the TMI, containing $3 d-, 4 s-$, and $4 p$-orbitals and to include one lone pair orbital per donor atom of the ligand thus giving the following picture of one-electronic states of the closest ligand shell (CLS) of a TMI in a TMC which the octahedral local symmetry:

$$
\begin{aligned}
& \psi^{a}\left(e_{g c}\right)=-x_{e_{g}} \phi\left(d_{z^{2}}\right)+\frac{y_{e_{g}}}{\sqrt{12}}\left(2 \chi_{z}+2 \chi_{-z}-\chi_{x}-\chi_{-x}\right. \\
& \left.-\chi_{y}-\chi_{-y}\right) \\
& \psi^{b}\left(e_{g c}\right)=y_{e_{g}} \phi\left(d_{z^{2}}\right)+\frac{x_{e_{g}}}{\sqrt{12}}\left(2 \chi_{z}+2 \chi_{-z}-\chi_{x}-\chi_{-x}\right. \\
& \left.-\chi_{y}-\chi_{-y}\right) \\
& \psi^{a}\left(e_{g s}\right)=-x_{e_{g}} \phi\left(d_{x^{2}-y^{2}}\right)+\frac{y_{e_{g}}}{2}\left(\chi_{x}+\chi_{-x}-\chi_{y}-\chi_{-y}\right) \\
& \psi^{b}\left(e_{g s}\right)=y_{e_{g}} \phi\left(d_{x^{2}-y^{2}}\right)+\frac{x_{e_{g}}}{2}\left(\chi_{x}+\chi_{-x}-\chi_{y}-\chi_{-y}\right)
\end{aligned}
$$




$$
\begin{aligned}
& \begin{aligned}
\psi^{a}\left(a_{1 g}\right)=x_{a_{1 g}} \phi(4 s)+\frac{y_{a_{1 g}}}{\sqrt{6}}\left(\chi_{x}+\chi_{y}+\chi_{z}+\chi_{-x}+\right. & \chi_{-y} \\
& \left.+\chi_{-z}\right)
\end{aligned} \\
& \begin{aligned}
& \psi^{b}\left(a_{1 g}\right)=y_{a_{1 g}} \phi(4 s)+\frac{x_{a_{1 g}}}{\sqrt{6}}\left(\chi_{x}+\chi_{y}+\chi_{z}+\chi_{-x}+\right.\left.\chi_{-y}\right) \\
& \psi_{-z}\left(t_{1 u \gamma}\right)=-x_{t_{1 u}} \phi\left(4 p_{\gamma}\right)+\frac{y_{t_{1 u}}}{\sqrt{2}}\left(\chi_{\gamma}-\chi_{-\gamma}\right)
\end{aligned} \\
& \psi^{b}\left(t_{1 u \gamma}\right)=y_{t_{1 u}} \phi\left(4 p_{\gamma}\right)+\frac{x_{t_{1 u}}}{\sqrt{2}}\left(\chi_{\gamma}-\chi_{-\gamma}\right) .
\end{aligned}
$$

The Stevens' coefficients $x_{\Gamma}, y_{\Gamma}$ subject to the normalization condition:

$$
x_{\Gamma}^{2}+y_{\Gamma}^{2}=1
$$

give the amount of the delocalization of the TMIcentered states and have to be determined (ideally) from a self-consistent field procedure applied to the effective Fock operator describing the CLS of the TMI. By this, the one-electron states of both the TMI and of the surrounding ligand atoms are explicitly included into consideration. In a symmetric environment assumed in Eq. (2), the $d$-orbitals of the $t_{2 g}$-symmetry neither get any admixture nor experience any change of their energy ( $\pi$-orbitals on the ligands are not taken into account at this point). The antibonding orbitals of the $e_{g}$-symmetry $\left(\psi^{a}\left(e_{g}\right)\right)$ are shifted upwards in energy:

$$
E_{e_{g}}^{*}=H_{d d}+\frac{H_{d \chi}^{2}}{H_{d d}-H_{\chi \chi}},
$$

where $H_{d d}, H_{\chi \chi}, H_{d \chi}$ are matrix elements of the one-electron Hamiltonian of the CLS, thus giving the following estimate for the splitting parameter:

$$
10 D q=\frac{H_{d x}^{2}}{H_{d d}-H_{\chi \chi}} .
$$

Within the LFT also only qualitative explanations could be obtained. It remained unclear where to get the values of $H_{\chi \chi}$ and $H_{d \chi}$ on which all the details of composition and structure of the ligands and their interaction are loaded in this model. Nevertheless, the expression Eq. (5) allows for important conclusions different from the ionic model of the CFT. Within the LFT, the splitting comes not from the (effective) charges localized on the ligands, but is a consequence of covalent interactions-ultimately of the interplay between the delocalization of the $d$-electronic states to the ligands and of the ligand electronic states to the $d$-shell. Spectrochemical series Eq. (1) then can be thought to be arranged in the order of increase of not only $10 \mathrm{Dq}$ but also of the characteristic delocalization parameters $x_{e_{y}}^{2} y_{e^{\prime}}^{2}$ describing the strength of the covalent metal-ligand interaction and determined by the same Hamiltonian matrix elements as the splitting through the relations:

$$
\begin{gathered}
x_{e_{g}}^{2} y_{e_{g}}^{2}=\frac{1}{4}\left(1-\frac{1}{1+\zeta_{e_{g}}^{2}}\right), \\
\zeta_{e_{g}}=\frac{H_{d \chi}}{H_{d d}-H_{\chi \chi}} .
\end{gathered}
$$

Getting very large $\zeta_{e_{g}}$ yields the highest possible value of $x_{e_{g}}^{2} y_{e_{g}}^{2}=1 / 4$ referring to the maximal possible delocalization of the $d$-orbital.

These considerations dating back to 30 s qualitatively explain pretty much of the spectroscopy of TMCs in terms of the $10 D q$ splitting parameter for the one-electron $d$-levels (although the consistent estimation of these and similar quantities through a kind of quantum chemical procedure-from the information on chemical composition and geometry of the TMC - was not possible).

At this point, one must say that the electronic structure of TMCs is much more sophisticated and does not reduce to the one-electronic states as presented by Eq. (2). The $d$-shells of the TMIs within the TMCs may to a large extent retain the multiplet structure they possess in the free state. In the free state, however, the excitation spectra of the $d$-shells, obviously, cannot have anything to do with the splitting and are controlled by the electron-electron interactions only. The characteristic parameters of that latter are known as the Slater-Condon parameters $F^{k}, k=0$, 2,4 , or the Racah parameters $A, B$, and $C$. It is remarkable that even in the simplest version of the CFT these quantities had to be taken into account (although considered as empirical parameters) to reproduce correct sequence of the many-electronic states of different total spin and spatial symmetries. Even more, in some situations it is possible exactly, although in others to a good approximation, to express the excitation energies in the $d$-shells of TMCs in terms of the electronelectron interaction (Slater-Condon or Racah) parameters only. In the course of attempts to fit the 
(appropriate) excitation energies to the models expressing them linearly through the Racah parameters, Jaurgensen (as well as other students of that time) noticed that the interaction parameters for the TMIs' $d$-shells in TMCs coming from such fits are systematically smaller than the same parameters derived from the spectra of the free ions. This phenomenon received the aforementioned beautiful Greek name (although well before similar, but much less pronounced behavior had been observed in lanthanide complexes and had the name of the Ephraim's effect) since it had been interpreted as a consequence of certain increase of the average electron-electron separation in the $d$ shell taking place due to the expansion of the latter in the course of the complex formation. Two mechanisms tentatively leading to the mentioned expansion had been discussed in Ref. [1].

First was the central field covalency (CFC) mechanism, which is based on the idea that the surrounding charges may contribute to the screening of the $d$-electrons they surround and by this may affect the rate of the radial decay of the $d$-states of a TMI in a TMC. It could, however, only happen if the spherical component of the external (ligand) Coulomb potential significantly depends on the distance in the $d$-shell region. Employing the wellknown expansion of the Coulomb potential over the spherical harmonics:

$$
\begin{aligned}
& \frac{1}{|\mathbf{R}-\mathbf{r}|} \\
& \quad=4 \pi \sum_{l=0}^{\infty} \frac{\min (r, R)^{l}}{\max (r, R)^{l+1}} \frac{1}{2 l+1} \sum_{m=-l}^{l} Y_{l m}^{*}\left(\frac{\mathbf{r}}{r}\right) Y_{l m}\left(\frac{\mathbf{R}}{R}\right)
\end{aligned}
$$

(here $R$ and $r$ are, respectively, the lengths of the radius vectors $\mathbf{R}$ and $\mathbf{r}$ ) shows that the spherically symmetric component $(l=0)$ does not depend on the electron position $\mathbf{r}$ provided it is located closer to the coordinate origin than the ligand atom at $\mathbf{R}$ $(R>r)$. Together with the assumption about the compactness of the $d$-shell ( $\sigma R \gg 1$, where $\sigma$ is the exponent of the Slater $d$-orbital) this allows one to exclude the CFC mechanism on the general theoretical grounds. The same reasoning explains why it is not possible to stabilize any bound state of an electron (e.g., in a vacancy in a crystal or in a "cavity" in a liquid) by external charges.

Second mechanism considered was the symmetry restricted covalency (SRC), which explained the orbital expansion by the formation of delocalized MOs of predominantly $d$-character, but having some contribution from the ligand states as stipulated by the LFT Eq. (2). Accepting the SRC mechanism has two important consequences. First is the possibility to ascribe different extent of expansion to different one-electron states in the $d$-shell.

This option was used up to full scale in Ref. [1] where three nephelauxetic ratios $\beta_{e e r} \beta_{e t}$, and $\beta_{t t}$ $\left(\beta_{33}, \beta_{35}\right.$, and $\beta_{55}$ in the original notation) had been introduced to represent the effect of the expansion of the orbitals in the $e$ - and $t_{2}$-manifolds of octahedral and tetrahedral complexes on the Coulomb interaction matrix elements involving the orbitals from them. The numerical fits of the experimental data reviewed in Ref. [1] allowed to conclude that in all cases the following relation holds:

$$
1>\beta_{t t}>\beta_{e t}>\beta_{e e}
$$

in a fair agreement with the qualitative picture of orbitals Eq. (2) according to which no delocalization (expansion) of the $t$-orbitals takes place (thus giving $\beta_{t t}=1$, which must be overridden by including $\pi$-overlap in the consideration) and the noticeable delocalization is expected only for the $e$-orbitals.

Second consequence is that the delocalization of the $d$-states due to mixing with the ligand states as the only source of the orbital expansion puts the observed renormalization of electron-electron interactions in the $d$-shells in TMCs in a strict relation with the crystal field splitting of the latter. Indeed, as one can see, the estimates of the nephelauxetic ratios accepted in Ref. [1] are

$$
\beta_{t t}=x_{t_{2 g}}^{4} ; \quad \beta_{e t}=x_{t_{2 g}}^{2} x_{e_{g}}^{2} ; \quad \beta_{e e}=x_{e_{g}}^{4} .
$$

Taking into account that

$$
x_{e_{g}}^{2} \approx 1-\frac{1}{4} \zeta_{e_{g}}^{2}
$$

one may expect that the nephelauxetic series (that of the ligands with decreasing $\beta$ 's) has to be similar to the spectrochemical series (that of increasing $10 D q$, see earlier). This, however, does not happen and the nephelauxetic series for ligands (i.e., for each fixed TMI) can be presented as follows $[1,5,6]$ (in order of increase of the amount of renormalization): 
TABLE I

The estimates of the nephlauxetic ratios as extracted from experimental data given in Refs. [1, 5, 6].

\begin{tabular}{|c|c|c|c|}
\hline & $\beta_{\mathrm{B}}=B / B_{\mathrm{O}}$ & $\beta_{\mathrm{C}}=C / C_{0}$ & $\gamma=C / B$ \\
\hline \multicolumn{4}{|c|}{$\mathrm{Mn}^{2+}, B_{0}=860, C_{0}=3850, \gamma_{0}=4.447$} \\
\hline $\mathrm{MnF}_{6}^{4-}$ & 0.819 & 0.948 & 5.186 \\
\hline $\mathrm{MnCl}_{6}^{4-}$ & 0.743 & 0.893 & 5.382 \\
\hline $\mathrm{MnCl}_{4}^{2-}$ & 0.612 & 0.920 & 6.736 \\
\hline $\mathrm{MnBr}_{4}^{2-}$ & 0.623 & 0.917 & 6.585 \\
\hline $\mathrm{Mn}\left(\mathrm{H}_{2} \mathrm{O}\right)_{6}^{2+}$ & 0.795 & 0.939 & 5.298 \\
\hline \multicolumn{4}{|c|}{$\mathrm{Fe}^{3+}, B_{0}=1015, C_{0}=4800, \gamma_{0}=4.729$} \\
\hline $\mathrm{FeF}_{6}^{3-}$ & 0.675 & 0.773 & 5.416 \\
\hline $\mathrm{FeCl}_{4}^{-}$ & 0.506 & 0.569 & 6.315 \\
\hline
\end{tabular}

Racah parameters $B$ and $C$ are in $\mathrm{cm}^{-1}$

$$
\begin{aligned}
\mathrm{F}^{-}<\mathrm{H}_{2} \mathrm{O}<\operatorname{urea}(\mathrm{O})<\mathrm{NH}_{3}<\mathrm{En}<\frac{1}{2} \mathrm{Ox}^{2-} \\
\\
\quad<\mathrm{NCS}^{-}<\mathrm{Cl}^{-} \\
<\mathrm{CN}^{-}<\mathrm{Br}^{-}<\mathrm{N}_{3}^{-}<\mathrm{I}^{-}<\mathrm{S}^{2-}<\mathrm{Se}^{2-}<\mathrm{Te}^{2-}
\end{aligned}
$$

which in many cases demonstrates not only deviations from the spectrochemical series Eq. (1) but even inversions: $\mathrm{F}^{-}$vs. $\mathrm{I}^{-} ; \mathrm{Cl}^{-}$vs. $\mathrm{Br}^{-}$, etc.

It is worth noticing that the numerical values of the renormalization constants (nephelauxetic ratios) $\beta_{\Gamma \Gamma}$, had been derived from the experiment under some additional assumptions. First, it had been assumed that the Racah parameters $B$ and $C$ are related by a fixed coefficient about four. It is true for the theoretical values of these parameters defined through the Slater-Condon parameters taken as integrals over Slater type $d$-orbitals:

$$
\begin{aligned}
& F_{2}^{\mathrm{th}}(d d)=\frac{5 \times 2093}{49 \times 76800} \sigma_{d} \\
& F_{4}^{\mathrm{th}}(d d)=\frac{9 \times 91}{441 \times 9216} \sigma_{d}
\end{aligned}
$$

( $\sigma_{d}$ is the orbital exponent for the Slater $d$-function). Then taking into account the definitions of the Racah parameters

$$
\begin{gathered}
B^{\text {th }}=F_{2}^{\text {th }}(d d)-5 F_{4}^{\text {th }}(d d) \\
C^{\text {th }}=35 F_{4}^{\text {th }}(d d)
\end{gathered}
$$

one arrives to:

$$
C^{\text {th }} / B^{\text {th }}=\frac{175}{44}=4-\frac{1}{44} \approx 3.977 .
$$

However, the basic assumption of the rigid ratio $F_{\text {th }}^{2} / F_{4}^{\text {th }}$ of $69-4 / 5$ following from Eq. (13) and leading to Eq. (15) does not take place even in free ions, and thus $F_{2}(d d)$ and $F_{4}(d d)$ must be treated as independent parameters and so must be considered $B$ and $C$. According to [6] the ratio $C_{0} / B_{0}$ where subscript 0 refers to the free ions is always larger than four and smaller than five. Also the assumption that $B$ and $C$ renormalize by the same nephelauxetic ratio throughout the complex formation is only true if the renormalization takes place according to the SRC mechanism. Using the data on simple $d^{5}$ complexes (those of $\mathrm{Mn}^{2+}$ and $\mathrm{Fe}^{3+}$ ) for which the Racah parameters can be directly extracted from $d-d$ excitation spectra, we establish the remarkable deviations from the assumption of the uniform renormalization of the Racah parameters $B$ and $C$ as shown in Table I. According to it the $B$ parameters are always strongly renormalized than the $C$ parameters leading to the $C / B$ ratios in the complexes to be systematically larger than five and sometimes to exceed six. Also, the complexes with very close values of $10 D q$ are known to give sometimes different values of renormalization constants indicating the absence of the direct relation between the amount of delocalization of the $d$-shell and the interaction renormalization in it.

All this calls for a new visit to this fascinating area of theoretical inorganic chemistry.

\section{Effective Hamiltonian of Crystal Field Theory}

As we mentioned previously despite the correct qualitative description of the spectrochemical series 
in the LFT, it cannot provide a quantitative method for calculation of the splittings of $d$-levels. The reasons were mentioned earlier-the unclear source of the matrix elements $H_{\chi \chi}$ and $H_{d \chi}$ to be used in Eq. (4) and the need to take into account the details of the electron-electron interaction (correlation) of electrons in the $d$-shells of TMIs. These deficiencies were the fundamental reason to develop the Effective Hamiltonian Crystal Field (EHCF) theory Ref. [7], whose basic purpose was to unite the estimate of the crystal field induced by the ligands with taking into account the correlations of electrons in the $d$-shells. This twofold task required the usage of a twofold tool: i.e., of combining the McWeeny [8] group function (GF) representation of the molecular electronic structure with the Löwdin partition technique Ref. [9]. The GF method as applied to TMCs assumes the wave functions of the ground and lower-energy excited states of the latter to have the form:

$$
\Psi_{n}=\Phi_{n}^{d} \wedge \Phi_{0}^{l}
$$

where $\Phi_{n}^{d}$ is some full CI function for $n_{d}$ electrons in the $d$-shell, and $\Phi_{0}^{l}$ is a single-determinant ground state for the $l$-system. Taking this way of segmenting the electronic structure into groups reflects the main feature of that of the TMCs, which is the presence of the strongly correlated $d$-shell of a specified composition, i.e., containing some fixed integer number $n_{d}$ of $(d$-)electrons with low energy excitations localized in it and of relatively inert (i.e., having rather high excitation energies) ligands. To be able to reproduce the splitting of the states of the $d$-shell under the influence of the $l$-system, the one electron transfers (delocalization) between the $d$ and $l$-systems have to be taken into account as in the LFT. Basically such transfers destroy the simple form of the wave function Eq. (16) by admixing the states with $n_{d} \pm 1\left(n_{l} \mp 1\right)$ electrons in the $d$ - and $l$-systems (charge transfer states) to it. However, experimentally we see that the number of electrons in the $d$-shell is a good quantum number and is perpetually used in chemical classification of the TMCs. Of course, there are situations when classification in terms of fixed number electrons in the $d$-shell fails, but we consciously exclude them from the current consideration. On the other hand, one has to realize that TMCs conforming to this classification do conform to it exactly, not approximately: their $d-d$-excitation spectra are precisely those of three or five electrons in this shell since there is no such a thing as a spectrum of approximately 5.1- or 3.2-electron system. Thus, in the compounds of interest it goes about some effective $d$-shell with the fixed number electrons in it. In such a setting, the contribution of the charge transfer states is taken into account with the use of the Löwdin projection technique. It is done as follows: the total Hamiltonian for a TMC is rewritten in the form:

$$
H=H_{d}+H_{l}+H_{c}+H_{r}
$$

where $H_{d}$ is the Hamiltonian for $d$-electrons in the field of the TMC atomic cores, $H_{l}$ is the Hamiltonian for the l-system electrons, $H_{c}$ and $H_{r}$ are, respectively, the operators of Coulomb and resonance interactions between two electron groups singled out in the TMC.

Projecting the exact Hamiltonian Eq. (17) is performed to the subspace spanned by the functions with the fixed number $n_{d}$ of $d$-electrons. By this the original Hamiltonian Eq. (17) is replaced by the effective one acting in this configuration subspace. By simple algebra the explicit form of the effective Hamiltonian is obtained [7]:

$$
\begin{aligned}
H^{\mathrm{eff}} & =P H_{0} P+H_{r r} \\
H_{0} & =H_{d}+H_{l}+H_{c} \\
H_{r r} & =P H_{r} Q\left(E Q-Q H_{0} Q\right)^{-1} Q H_{r} P
\end{aligned}
$$

where $P$ is the operator projecting to the subspace of the functions with the fixed number $n_{d}$ of the $d$-shell electrons and $n_{l}=N-n_{d}$ is that of the l-subsystem electrons; and $Q=1-P$.

The eigenstate energies must be obtained from the relation:

$$
E_{n}=\left\langle\Phi_{n}\left|H^{\mathrm{eff}}\left(E_{n}\right)\right| \psi_{n}\right\rangle .
$$

Eigenvalues of the effective Hamiltonian coincide with exact Hamiltonian eigenvalues by construction. Since the effective Hamiltonian depends on energy, the last equation must be solved iteratively until convergence in energy is achieved. However, since the charge transfer states (determining the poles of the above effective Hamiltonian) lay significantly higher in energy than the $d$-shell excitations this dependence turns out to be weak and can be neglected, so one can set:

$$
H^{\mathrm{eff}}\left(E_{n}\right) \approx H^{\mathrm{eff}}\left(E_{0}\right)
$$


where $E_{0}$ is the ground state energy of the Hamiltonian $\mathrm{H}_{0}$. Thus obtained effective Hamiltonian corresponds to the second order of the Raleigh-Schrödinger perturbation theory in $H_{r}$.

Variation principle applied to the effective Hamiltonian with the trial function of the form Eq. (16) leads to the self-consistent system of equations:

$$
\begin{aligned}
H_{d}^{\mathrm{eff}} \Phi_{n}^{d} & =E_{n}^{d} \Phi_{n}^{d} \\
H_{l}^{\mathrm{eff}} \Phi_{0}^{l} & =E_{0}^{l} \Phi_{0}^{l} \\
H_{d}^{\mathrm{eff}} & =H_{d}+\left\langle\Phi_{0}^{l}\left|H_{c}+H_{r r}\right| \Phi_{0}^{l}\right\rangle, \\
H_{l}^{\mathrm{eff}} & =H_{l}+\left\langle\Phi_{0}^{d}\left|H_{c}+H_{r r}\right| \Phi_{0}^{d}\right\rangle .
\end{aligned}
$$

In this system, the effective Hamiltonian $H_{d}^{\text {eff }}$ for the $d$-electron subsystem depends on the wave function of the ligand subsystem $\Phi_{0}^{l}$, and in its turn the effective Hamiltonian $H_{l}^{\text {eff }}$ for the ligand subsystem depends on the $d$-electrons' wave functions $\Phi_{0}^{d}$. These equations must be solved self-consistently as well. In the EHCF method, Ref. [7], the Slater determinant $\Phi_{0}^{l}$ is constructed of MO's of the $l$-system, obtained from the Hartree-Fock equations in the $\mathrm{CNDO} / 2$ approximation for the valence electrons of the ligands. In this case, the transition from the bare Hamiltonian $H_{l}^{\text {eff }}$ for the $l$-system to the corresponding effective (dressed) Hamiltonian reduces to renormalization of one-electron parameters related to the TMI:

$$
\begin{gathered}
U_{i i}^{\text {eff }}=U_{i i}+\frac{1}{5} n_{d} \sum_{\mu} g_{\mu i} \\
Z_{M}^{\text {eff }}=Z_{M}-n_{d},
\end{gathered}
$$

where $U_{i i}$ is the parameter of the interaction of $4 s-$ and $4 p$-electrons $\left(i=4 s, 4 p_{x}, 4 p_{y}, 4 p_{z}\right)$ with the TMI core, $Z_{M}$ is TMI core charge, $g_{\mu i}=(\mu \mu \mid i i)-1 / 2$ $(\mu i \mid i \mu)$ are the parameters of intraatomic Coulomb interactions. The $\Phi_{0}^{l}$ function thus obtained is used further for constructing the effective Hamiltonian for the $d$-shell.

The effective Hamiltonian for the $d$-shell after averaging Eq. (21) inter-subsystem interaction operators $H_{c}$ and $H_{r r}$ over the ground state of the l-system takes the form:

$H_{d}^{\mathrm{eff}}=\sum_{\mu \nu \sigma} U_{\mu \nu}^{\mathrm{eff}} d_{\mu \sigma}^{+} d_{\nu \sigma}+\frac{1}{2} \sum_{\mu \nu \rho \eta \sigma \tau} \sum_{\sigma \tau}(\mu \nu \mid \rho \eta) d_{\mu \sigma}^{+} d_{\rho \tau}^{+} d_{\eta \tau} d_{\nu \sigma}$ where $d_{\mu \sigma}^{+}\left(d_{\nu \sigma}\right)$ are operators of creation (annihilation) of electron with the spin projection $\sigma$ on the $\mu$ th $d-\mathrm{AO} ;(\mu \nu \mid \rho \eta)$ are the two-electron integrals of the Coulomb interaction in the $d$-shell. Effective one-electron parameters $U_{\mu \nu}^{\text {eff }}$ of the $d$-shell contain contributions from the Coulomb and from the projected [Eq. (5)] resonance interaction with the $l$ system:

$$
U_{\mu \nu}^{\mathrm{eff}}=\delta_{\mu \nu} U_{d d}+W_{\mu \nu}^{\mathrm{atom}}+W_{\mu \nu}^{\mathrm{ion}}+W_{\mu \nu}^{\mathrm{cov}},
$$

where

$$
\begin{gathered}
W_{\mu \nu}^{\text {atom }}=\delta_{\mu \nu}\left(\sum_{i \in s, p} g_{\mu, i} P_{i i}\right) \\
W_{\mu \nu}^{\text {ion }}=\sum_{L}\left(P_{L L}-Z_{L}\right) V_{\mu \nu}^{L} .
\end{gathered}
$$

Here $P_{i i}$ is one-electron density matrix element for the ligand subsystem, $P_{L L}=\Sigma_{l \in L} P_{l l}, Z_{L}$ is the $L$ th atom core charge, $V_{\mu \nu}^{L}$ is the matrix element of the $d$-electron potential energy in the electrostatic field of a unit point charge placed on the $L$ th ligand atom (see later). The covalence contribution to the crystal field is given by

$$
W_{\mu \nu}^{\mathrm{cov}}=-\sum_{i}^{(\mathrm{MO})} \beta_{\mu i} \beta_{\nu i}\left\{\frac{1-n_{i}}{\Delta E_{d i}}-\frac{n_{i}}{\Delta E_{i d}}\right\}
$$

where $\beta_{\mu i}$ is the one-electron matrix element (resonance or hopping integral) between the $\mu$ th $d$-orbital and the ith ligand $\mathrm{MO}, n_{i}(=0,1)$ is the occupation number of the $i$ th $\mathrm{MO}, \Delta E_{d i}\left(\Delta E_{i d}\right)$ are excitation energies required to transfer an electron from the $d$-shell (ith $\mathrm{MO}$ ) to the ith $\mathrm{MO}$ ( $d$-shell).

The effective Hamiltonian for the $d$-shell Eq. (23) formally coincides with the CFT Hamiltonian. The substantial difference is the covalence contribution Eq. (26) to the $d$-shell one-electron parameters Eq. (24), taking into account the effect of virtual charge transfers between the metal $d$-shell and the ligands, i.e., the delocalization of the $d$-states. Thereby, the EHCF contains not only electrostatic but also covalence terms coming from the resonance interactions between the $d$-shell and the ligands as the LFT does.

According to calculations performed in Refs. [7, 10-13] for the TMCs of divalent cations, the covalence contribution to the splitting parameter $10 \mathrm{Dq}$ dominates and gives up to $90 \%$ of the total. This allows to state that like in the LFT in the EHCF, the splitting of the $d$-shell is controlled by an analog of 
the above $\zeta$ parameter, i.e., by a ratio of the electron hopping integral to the energy of the inter-subsystem charge transfer state. Generally, the EHCF method allowed to describe correctly the symmetry of the ground states and the optical $d-d$-transition energies with precision up to $1000 \mathrm{~cm}^{-1}$ for about a hundred of the TMCs of the first transition row divalent cations ranging from hexafluoroanions to porphyrine complexes and improved the semiempirical description of TMC electronic structure significantly.

\section{Renormalization of Electron-Electron Interaction in the d-Shell}

The EHCF method as presented in the previous section lacks an important element: no renormalization of the electron-electron interaction in the $d$ shell appears from the above derivation. Strict application of the EHCF theory assumes that the free ion values $B_{0}$ and $C_{0}$ of the Racah parameters are used. This, however, would contradict to the experiment and thus the experimental values of $B$ and $C$ had been used throughout the calculations in Refs. $[7,10-13]$. This clearly reduces the predictive power of the otherwise very attractive EHCF picture and of the pragmatic calculation method based upon it. For that reason, we undertake this study in which we try to obtain the estimate of the amount of the renormalization of the electron-electron interactions in the $d$-shell within the same framework, which previously allowed very precise estimates of the splitting of the $d$-shell. Before doing so, we notice that these two effects: splitting and interaction renormalizations appear in different orders of perturbation theory with respect to one-electron hopping matrix element responsible for charge transfers between the $d$ - and $l$-systems. The splitting is of the second order in $\zeta$ whereas renormalization must be of the fourth order. ${ }^{1}$ This also agrees with the LFT estimates Eq. (10) and explains our old result where only the aforementioned perturbational estimate of the fourth order had been tested ${ }^{2}$ and the nephelauxetic ratios turned out to be ca. $0.98 \div 0.99$ in all considered cases. This failure

\footnotetext{
${ }^{1}$ It is worth noting that the very notation of in the theory of the nephelauxetic effect is not particularly convenient. The quantities of definite order are not the nephelauxetic ratios themselves $\beta$, but their deviations from the unity: $1-\beta$.

${ }^{2}$ Kharitonov, D. N.; Tchougréeff, A. L. unpublished.
}

indicates that in the EHCF context some other reasons of the renormalization of the Coulomb interaction between electrons in the $d$-must be admitted.

\subsection{CONTINOUS INSULATOR MODEL OF NEPHELAUXETIC EFFECT}

It is fair to say that the ligand polarization had been mentioned as a mechanism of renormalization of Coulomb interaction in the $d$-shells of TMCs at a pretty early stage. If the nephelauxetic series Eq. (12) is rewritten in terms of donor atoms Ref. [5], it becomes:

$$
\mathrm{F}<\mathrm{O}<\mathrm{N}<\mathrm{Cl}<\mathrm{Br}<\mathrm{I}<\mathrm{Se}<\mathrm{Te}
$$

which approximately corresponds to the order of the atomic polarizabilities. It, however, had been considered as a secondary effect in Ref. [1], although obvious deviations between the spectrochemical and nephelauxetic series were clear. The same idea had been used in Ref. [14]. According to these authors, if one assumes that a TMI bearing the $d$-shell with the Slater orbital exponent $\sigma_{d}$ is placed inside a sphere of radius $b$ cut in the medium with dielectric constant, $\varepsilon$, the Slater-Condon parameters $F_{2}(d d)$ and $F_{4}(d d)$ renormalize with the nephelauxetic ratios $\beta_{2}$ and $\beta_{4}$ given by:

$$
\begin{aligned}
& 1-\beta_{2}=\frac{1260}{299}\left(\frac{\varepsilon-1}{3 \varepsilon+2}\right)\left(\frac{4}{b \sigma_{d}}\right)^{5} \\
& 1-\beta_{4}=\frac{20 \times 10 !}{13}\left(\frac{\varepsilon-1}{5 \varepsilon+4}\right)\left(\frac{1}{b \sigma_{d}}\right)^{9} \\
& \frac{1-\beta_{2}}{1-\beta_{4}}=\frac{4}{5175}\left(\frac{5 \varepsilon+4}{3 \varepsilon+2}\right)\left(b \sigma_{d}\right)^{4} .
\end{aligned}
$$

Assuming $\varepsilon=12, b=1.5 \AA$, and $\sigma_{d}=2.06$, the authors in Ref. [14] reproduced the magnitude of the $\beta_{4}$ ratio for one particular case $\left(\mathrm{Co}^{2+}\right.$ impurity ion in the tetrahedral site of $\mathrm{MgAl}_{2} \mathrm{O}_{4}$ ) and underestimated $\beta_{2}$ by ca. $40 \%$, thus yielding the correct order of magnitude of the effect. The approximation of infinite space filled by an insulator outside the sphere reasonable in the case of a impurity TMI in the crystals is not that much important since obvious transition to the model of a spherical layer reduces to subtracting of corresponding powers of $b^{*} \sigma$ ( $b^{*}$ is the external radius of the spherical layer) does not significantly affect the numerical result due to high powers of the $b / b^{*}<1$ ratio as entering in the correcting multipliers $\left(1-\left(b / b^{*}\right)^{5}\right)$ and $(1-$ 
$\left.\left(b / b^{*}\right)^{9}\right)$ for the first two rows of Eq. (27). One can estimate to what extent the value of $\varepsilon=12$ used in Ref. [14] can be reproduced by available data on polarizability of the ligands using the ClausiusMossotti formula for $\varepsilon$ :

$$
\varepsilon=1+\frac{4 \pi}{3} \rho \alpha .
$$

According to Ref. [1], they range from ca. $1 \AA^{3}$ for $\mathrm{F}^{-}$to $14 \div 16 \AA^{3}$ for $\mathrm{Te}^{2-}$, whereas the densities to be used to get the above value of the dielectric constant range from unrealistically high value of 2.5 ions per $\AA^{3}$ for $\mathrm{F}^{-}$to unrealistically low 0.16 ions per $\AA^{3}$ for $\mathrm{Te}^{2-}$. These estimates show that the polarizability-based model of renormalization of the Coulomb interaction of $d$-electrons can cover a wide range of observed values of renormalization. Remarkably enough the formulae Eq. (27) have the multiplicative structure

$$
1-\beta \approx h(\text { ligands }) k(\text { metal })
$$

prescribed by Ref. [1] with $h$ to be identified with the $\varepsilon$-dependent multiplier and $k$ to be the $\sigma_{d}$-dependent one.

\subsection{POLARIZATION PROPAGATOR MODEL OF NEPHELAUXETIC EFFECT}

From the quantum chemical point of view, the expression Eq. (27) lacks the atomic (or even orbital) resolution necessary for quantum chemistry. To derive the analog of the result, Eq. (27) compatible with quantum chemical description and particularly with the EHCF theory one has to follow the general theory of the GF-based semiempirical methods as described in Refs. [15-17].

It must be noticed that the derivation of EHCF was performed in an assumption that not only the charge transfer excitations in the $n_{d} \pm 1\left(n_{l} \mp 1\right)$ electrons manifold have the energy large enough to assure the validity of the perturbation theory with respect to small parameter $\zeta$ but also the energies of the excitations in the $l$-system are large enough not to take them into account. The excitations of the $l$-system are, however, responsible for its polarization. One has to realize that taking the wave function of the TMC in the form of Eq. (16) even after Löwdin partition with use of the complementary projection operators $P$ and $Q$ is an approximation. In fact, the operator $P$ projects out the states with the charge transfer between the $d$ - and $l$-system, but does not assure the product form of Eq. (16). Products of different states of the $d$ - and $l$-systems satisfying only the condition of the fixed number of the electrons in each of the singled out groups enter in the expansion of the true ground state of the effective Hamiltonian Eq. (18). The form Eq. (16) is a kind of self-consistent field approximation to it. To improve this description one has to perform one more projection namely to the subspace of the products where all possible states of the $l$-system are replaced by its ground state. Formally, we previously consider the projection operator $\mathscr{P}$ :

$$
\mathscr{P}=I_{d} \otimes\left|\Phi_{0}^{l}\right\rangle\left\langle\Phi_{0}^{l}\right|,
$$

and the complementary projection operator $Q=$ $1-\mathscr{P}$ on the subspace orthogonal to it. Inserting the projection operators $\mathscr{P}$ and $Q$ into general expressions for the effective Hamiltonian acting in the subspace Im $\mathscr{P}$ (spanned by the functions of the form Eq. (16)-all possible $\Phi_{n}^{d}$ times the ground state $\left.\Phi_{0}^{l}\right)$, yields the expression:

$$
\begin{gathered}
H^{\mathrm{eff}}(\omega)=\mathscr{P} H^{\mathrm{eff} \mathscr{P}}+\mathscr{P} H^{\mathrm{eff}} Q R(\omega) Q H^{\mathrm{eff}} \mathscr{P}, \\
R(\omega)=\left(\omega Q-Q H^{\mathrm{eff}} Q\right)^{-1} .
\end{gathered}
$$

The first term is precisely $d$-electron Hamiltonian Eq. (23) multiplied by the l-system ground-state projection operator $\left|\Phi_{0}^{l}\right\rangle\left\langle\Phi_{0}^{l}\right|$. The second gives the correction to it. Different terms in $H^{\text {eff }}$ behave differently under this projection. Taking into account that $\mathscr{P}$ projects to the product of the eigenstates of the operators $H_{d}^{\text {eff }}$ and $H_{l}^{\text {eff }}$ Eq. (21) one can see that:

$$
P H^{\mathrm{eff}} Q=P W_{d l} Q,
$$

where

$$
W_{d l}=\hat{W}_{d l}-\left\langle\left\langle\hat{W}_{d l}\right\rangle\right\rangle_{d}-\left\langle\left\langle\hat{W}_{d l}\right\rangle\right\rangle_{l}
$$

is the operator of reduced interaction of the $d$ - and l-systems where:

$$
\begin{gathered}
W_{d l}=V^{c}+V^{r r} \\
V^{c}=H^{c}-\left\langle\left\langle H^{c}\right\rangle\right\rangle_{d}-\left\langle\left\langle H^{c}\right\rangle\right\rangle_{l} \\
V^{r r}=H^{r r}-\left\langle\left\langle H^{r r}\right\rangle\right\rangle_{d}-\left\langle\left\langle H^{r r}\right\rangle\right\rangle_{l}
\end{gathered}
$$

where the expectation values of the interaction operators calculated for the ground state of the $d$ - and 
l-systems are subtracted (i.e., only the fluctuations of the interacting quantities are retained). With these notions, the correction acquires the form:

$$
\mathscr{P} W_{d l} \mathscr{R}(\omega) W_{d l} \mathscr{P} .
$$

As before the idea of relative inertness of the $l$-system is formalized by the assumption that the excitation energies in it are large as compared with the excitation energies in the $d$-system, which are only interesting for us. For this reason, one can guess that the dependence of the resolvent on $\omega$ is weak and that the values of $\omega$ in the interesting energy range are much smaller than the resolvent poles that are all lying not lower than the first excitation energy in the $l$-system. These notions allow to replace the resolvent $R(\omega)$ by its value at $\omega$ $=0$ (by this, the electronic dynamic effects in the $l$-system are excluded):

$$
\mathscr{R}(0)=-\sum_{\lambda \neq 0} \frac{\left|\Phi_{\lambda}^{l}\right\rangle\left\langle\Phi_{\lambda}^{l}\right|}{E_{\lambda}^{l}},
$$

where $E_{\lambda}^{l}$ are excitation energies in the $l$-system. Restricting for the simplicity in Eq. (32) by the reduced Coulomb operator:

$$
\begin{aligned}
& V^{c}=\sum_{\substack{d d^{\prime} \in d \\
l l^{\prime} \in l}}\left(d d^{\prime} \mid l l^{\prime}\right)\left[d^{+} d^{\prime} l^{+} l^{\prime}-l^{+} l^{\prime}\left\langle\left\langle d^{+} d^{\prime}\right\rangle\right\rangle_{d}\right. \\
&\left.-d^{+} d^{\prime}\left\langle\left\langle l^{+} l^{\prime}\right\rangle\right\rangle_{l}\right],
\end{aligned}
$$

we get after averaging over the ground state of the $l$-system the following correction to the $d$-system Hamiltonian:

$$
\sum_{d d^{\prime} d^{\prime \prime} d^{\prime \prime \prime} \in d} d^{+} d^{\prime} d^{\prime \prime} d^{\prime \prime \prime} \sum_{l l^{\prime} l^{\prime} l^{\prime \prime} \in l}\left(d d^{\prime} \mid l l^{\prime}\right)\left(l^{\prime \prime} l^{\prime \prime \prime} \mid d^{\prime \prime} d^{\prime \prime \prime}\right) \prod_{l l^{\prime} l^{\prime} l^{\prime \prime}}^{l}(0),
$$

describing the weakening of the interaction between the fluctuations of the electron density in the $d$-shell due to interaction between the polarizations induced by these fluctuations in the l-system. The polarization propagator of the $l$-system entering the answer is:

$$
\Pi_{l l^{\prime} l^{\prime} l^{\prime \prime}}^{l}(\omega)=\sum_{\lambda \neq 0}\left\langle\Phi_{0}^{l}\left|l^{+} l^{\prime}\right| \Phi_{\lambda}^{l}\right\rangle\left(\omega-E_{\lambda}^{l}\right)^{-1}\left\langle\Phi_{\lambda}^{l}\left|l^{\prime \prime+} l^{\prime \prime \prime}\right| \Phi_{0}^{l}\right\rangle .
$$

The result Eq. (36) can be rewritten in terms of the polarization correction to the two-electron matrix elements:

$$
\delta\left(d d^{\prime} \mid d^{\prime \prime} d^{\prime \prime \prime}\right)=\sum_{l l^{\prime} l^{\prime \prime} l^{\prime \prime} \in l}\left(d d^{\prime} \mid l l^{\prime}\right) \Pi_{l l^{\prime} l^{\prime \prime} l^{\prime \prime \prime}}\left(l^{\prime \prime} l^{\prime \prime \prime} \mid d^{\prime \prime} d^{\prime \prime \prime}\right) .
$$

Significant simplification can be reached in the basis of canonical MOs of the $l$-system where the polarization propagator is diagonal in that sense that only the matrix elements of the form $\Pi_{l l^{\prime} l^{\prime} l}$ are nonvanishing where $l$ and $l^{\prime}$ refer to the occupied and vacant states, respectively, of the $l$-system.

The correction Eq. (36) must be included if the fluctuation of the electronic density in the $l$-system of the TMC as compared with the wave function obtained as solution of the system Eq. (21) are not considered explicitly. This is precisely the case in the EHCF theory. Incidentally, the intrashell SlaterCondon parameters $F^{k}(d d)$ with $k=2,4$ are precisely those which describe the interactions between the density fluctuations in the $d$-shell and those which one should expect to renormalize to reproduce the nephelauxetic effect. The Hamiltonian matrix elements capable to couple the density fluctuations in the $d$-shell with those in the surrounding are those of the Coulomb interaction. In the free ion, the fluctuation operators like $d^{+} d^{\prime}$ transform according to the $\mathrm{SO}(3)$ representations characterized by the angular momentum 2 and 4 , which are incidentally used for classification of the Slater-Condon parameters.

As it is shown in Ref. [7], the matrix elements of the form $\left(d d^{\prime} \mid l l^{\prime}\right)$ describing the Coulomb interaction between the electrons in the $d$ - and $l$-systems fall into two types (i) intraatomic ones, describing the interaction between the $d$ - and sp-shells of the TMI and (ii) those between the $d$-shell of the TMI and the electrons in the ligand atoms. In the ionic model of the CFT, the elements of the type (ii) are responsible for splitting of the $d$-levels. Accordingly, the Coulomb interaction operator $H^{c}$ is a sum:

$$
H^{c}=H_{1}^{c}+H_{2}^{c}
$$

and we consider the effect of two contributions to Eqs. (36)-(38) separately.

\subsubsection{Intraatomic Contribution to Nephelauxetic Effect}

In the intraatomic context, it is convenient to use the one-electron wave functions of definite angular 
momentum projection (complex spherical harmonics) instead of cubic harmonics usual for the atomic states in quantum chemistry. We start from reminding the form of a general intraatomic matrix elements of the Coulomb interaction between electrons. In general, for the atomic states $n_{i} l_{i} m_{i}, i=1 \div$ $4 \div n_{i}$ 's are the principal quantum numbers, $l_{i}$ 's are the azimuthal quantum numbers, and $m_{i}^{\prime}$ 's are magnetic quantum numbers of the states involved) the matrix element $\left(m_{1} m_{2} \mid m_{3} m_{4}\right)$ with $m_{1}=q-m_{2} ; m_{3}$ $=q+m_{4}$ and with respective $n_{i} l_{i}$ 's reads:

$$
\begin{array}{r}
(-1)^{q+l_{1}+l_{3}-\left(m_{2}+m_{4}\right)}\left(\begin{array}{ccc}
l_{1} & k & l_{2} \\
0 & 0 & 0
\end{array}\right)\left(\begin{array}{ccc}
l_{3} & k & l_{4} \\
0 & 0 & 0
\end{array}\right) \times \\
\times \sqrt{\left(2 l_{1}+1\right)\left(2 l_{2}+1\right)\left(2 l_{3}+1\right)\left(2 l_{4}+1\right)} \\
\times\left(\begin{array}{ccc}
l_{1} & k & l_{2} \\
q-m_{2} & -q & m_{2}
\end{array}\right) \\
\times\left(\begin{array}{ccc}
l_{3} & k & l_{4} \\
-\left(q+m_{4}\right) & q & m_{4}
\end{array}\right) R^{k}\left(\left\{n_{i} l_{i}\right\}\right)
\end{array}
$$

where the symbols $\left(\begin{array}{lll}\ldots & \ldots & \ldots \\ \ldots & \ldots & \ldots\end{array}\right)$ are the Wigner $3 j m$-symbols as described in many places, e.g., in Ref. [18]. For the interaction matrix elements in the $d$-shell it yields:

$$
\begin{aligned}
\left(m_{2}-q, m_{2} \mid m_{4}+q, m_{4}\right)=25 \sum_{k}(-1)^{q-\left(m_{2}+m_{4}\right)} \\
\times\left(\begin{array}{lll}
2 & k & 2 \\
0 & 0 & 0
\end{array}\right)^{2}\left(\begin{array}{ccc}
2 & k & 2 \\
q-m_{2} & -q & m_{2}
\end{array}\right) \\
\times\left(\begin{array}{ccc}
2 & k & 2 \\
-\left(q+m_{4}\right) & q & m_{4}
\end{array}\right) F^{k}(d d) .
\end{aligned}
$$

Intraatomic matrix elements coupling the fluctuations in the $d$ - and $p$-shells are those coupling the fluctuations with $k=2$ (for two $p$-functions $k=4$ is not accessible, whereas $k=0$ are not the fluctuations). Since in the octahedral and tetrahedral complexes the $p$-shell of the TMI remains nonsplitted (as in the free ion), the spherical harmonics can also be used for it. Thus we get:

$$
\begin{aligned}
&\left(d d^{\prime} \mid p p^{\prime}\right)=\left(m_{2}-q, m_{2} \mid m_{3}+q, m_{3}\right)==-15 \cdot \\
&(-1)^{q-\left(m_{2}+m_{3}\right)}\left(\begin{array}{ccc}
2 & 2 & 2 \\
0 & 0 & 0
\end{array}\right)\left(\begin{array}{ccc}
1 & 2 & 1 \\
0 & 0 & 0
\end{array}\right) \times \\
& \times\left(\begin{array}{ccc}
2 & 2 & 2 \\
q-m_{2} & -q & m_{2}
\end{array}\right) \\
& \times\left(\begin{array}{ccc}
1 & 2 & 1 \\
-\left(q+m_{3}\right) & q & m_{3}
\end{array}\right) F^{2}(p d) .
\end{aligned}
$$

A product of two such elements (to be further multiplied by the by the polarization propagator matrix element) is:

$$
\left(d d^{\prime} \mid p p^{\prime}\right)\left(p^{\prime} p \mid d^{\prime \prime} d^{\prime \prime \prime}\right) .
$$

Taking into account that the element $\left(p^{\prime} p \mid d^{\prime \prime} d^{\prime \prime \prime}\right)$ is as well proportional to $F^{2}(p d)$ with the symmetry multiplier

$$
\begin{aligned}
&\left(p^{\prime} p \mid d^{\prime \prime} d^{\prime \prime \prime}\right)=\left(m_{5}-p, m_{5} \mid m_{4}+p, m_{4}\right)= \\
&=15 \sum_{k}(-1)^{p+3-\left(m_{5}+m_{4}\right)}\left(\begin{array}{ccc}
2 & 2 & 2 \\
0 & 0 & 0
\end{array}\right)\left(\begin{array}{lll}
1 & 2 & 1 \\
0 & 0 & 0
\end{array}\right) \times \\
& \times \\
& \quad\left(\begin{array}{ccc}
2 & 2 & 2 \\
-\left(p+m_{4}\right) & p & m_{4}
\end{array}\right) \\
& \quad \times\left(\begin{array}{ccc}
1 & 2 & 1 \\
p-m_{5} & -p & m_{5}
\end{array}\right) F^{2}(p d)
\end{aligned}
$$

subject to the selection rules

$$
\begin{gathered}
m_{5}=q+m_{3} \\
m_{3}=-p+m_{5}
\end{gathered}
$$

which immediately yields

$$
p=q
$$

(transferred angular momentum projections must be equal). Using once again the first of the aforementioned conditions, we arrive to the following combination of the 3jm symbols depending on the momentum projections of the individual states in the $d$ - and the $p$-shells:

$$
\begin{aligned}
& (-1)^{q-\left(m_{2}+m_{4}\right)}\left(\begin{array}{ccc}
2 & k & 2 \\
q-m_{2} & -q & m_{2}
\end{array}\right) \\
& \times\left(\begin{array}{ccc}
2 & k & 2 \\
-\left(q+m_{4}\right) & q & m_{4}
\end{array}\right) \times \\
& \times\left(\begin{array}{ccc}
1 & 2 & 1 \\
-\left(q+m_{3}\right) & -q & m_{3}
\end{array}\right)\left(\begin{array}{ccc}
1 & 2 & 1 \\
-\left(q+m_{3}\right) & q & m_{3}
\end{array}\right) .
\end{aligned}
$$

First two multipliers and the phase factor yield precisely that of the matrix element $\left(m_{2}-q m_{2} \mid q+\right.$ $\left.m_{4} m_{4}\right)$ in the $d$-shell. Different values of $m_{3}$ in the second pair of multipliers number different (degen- 
erate) states in the $p$-shell, contributing to the renormalization. These products must be summed up which yields a factor of $1 / 5$ independent on $q$. We see that all matrix elements in the $d$-shell renormalize accordingly so that the result can be represented as a renormalization of the Slater-Condon parameter $F^{2}(d d)$. The correction it receives reads:

$$
-\frac{9}{5}\left(\begin{array}{lll}
1 & 2 & 1 \\
0 & 0 & 0
\end{array}\right)^{2}\left[F^{2}(p d)\right]^{2} \frac{x_{t_{11}}^{2} y_{t_{1 u}}^{2}}{\Delta_{t_{1 u}}^{2}}
$$

which after inserting the value of the $3 j m$ symbol reduces to

$$
-\frac{6}{25}\left[F^{2}(p d)\right]^{2} \frac{x_{t_{1 u}}^{2} y_{t_{1 u}}^{2}}{\Delta_{t_{1 u}}}
$$

In these expressions, the last multipliers represent the contribution of the CLS as defined in Ref. [19] to the polarization propagator of the $l$-system; $\Delta_{t_{11}}$ is the energy gap between the occupied and empty states of the $t_{1 u}$-symmetry spanned by the $p$-states of the TMI, and the $x_{t_{1 u}}$ and $y_{t_{11}}$ are the expansion coefficients of the corresponding MOs as defined by Eq. (2).

Already this result allows one to see that the renormalization of the intrashell interaction is not uniform in that sense that the proposed treatment renormalizes only the Slater-Condon parameter $F^{2}(d d)$, but not $F^{4}(d d)$. Thus in terms of the Racah parameters, it means that the $B$ parameter renormalizes whereas the $C$ parameter does not. That is what we could expect on the basis of our analysis summarized in Table I where the renormalization of the parameter $B$ is systematically much more pronounced than that of $C$. Of course one should not ascribe all this difference to the above Eq. (51) at least for two reasons: first or all the renormalization of $C$ is an established fact, which needs to be explained, and second, the estimate of $1-\beta_{2}$ coming from:

$$
\frac{6}{25} x_{t_{1 u}}^{2} y_{t_{1 u}}^{2} \frac{F^{2}(p d)}{F^{2}(d d)} \frac{F^{2}(p d)}{\Delta_{t_{1 u}}}
$$

turns out to be too small. Indeed, the ratio $F^{2}(p d) /$ $F^{2}(d d)$ of the "experimental" values falls in the range $0.1 \div 0.3$ for all TMIs of the first row. That calculated for the ratio $\sigma_{p} / \sigma_{d} \approx 0.5$ characteristic for the Burns' exponents used in the EHCF yields the ratio $F^{2}(p d) / F^{2}(d d)$ to be ca. $0.25 .{ }^{3}$ Nevertheless, the earlier formula has at least the use that it demonstrates the chemical specificity of the interaction renormalization and describes the intraatomic contribution to it which clearly cannot be covered by the continuous insulator model Eq. (27).

In more general setting not limited by the CLS model of the ligands, rather including all their valence orbitals like it is normally done in the EHCF Eq. (52) must be modified to

$$
1-\beta_{2}=\frac{6}{25} \frac{\left[F^{2}(p d)\right]^{2}}{F^{2}(d d)} \sum_{\gamma \in x, y, z, z \in \in \text { occ }} \sum_{\substack{i, j \\ \varepsilon_{j}}} \frac{\left(c_{i \gamma} c_{j \gamma}\right)^{2}}{\varepsilon_{i}}
$$

where the last sum is nothing but the orbital-orbital polarizability of the $\gamma$ th (4) $p$-AO of the TMI. Sensitivity to the chemical specific of the surrounding appears due to the $\mathrm{MO}$ coefficients of the respective $p$-AOs in the occupied and vacant MOs.

\subsubsection{Ionic Contribution to Crystal Field and the Nephelauxetic Effect}

Further and probably dominating contributions to the renormalization of the interaction parameters of the $d$-shell comes from the second (interatomic) term of the Coulomb interaction operator between the $d$ - and $l$-systems. As previously, the key element of the construction is identifying the matrix elements of the interaction which are responsible for the coupling between the density fluctuations in the corresponding systems. Incidentally, the EHCF contains the electrostatic interaction between the electrons in the $d$ - and the $l$-systems of the form:

$$
\begin{gathered}
H_{2}^{c}=\sum_{m m^{\prime}} \hat{V}_{m m^{\prime}} \\
\hat{V}_{m m^{\prime}}=\sum_{\substack{L \in L \\
\sigma, \tau}} \sum_{m m^{\prime},}^{L} d_{m \sigma}^{+} d_{m^{\prime} \sigma} l_{\tau}^{+} l_{\tau}
\end{gathered}
$$

with

\footnotetext{
${ }^{3}$ In fact, the ratio $F^{2}(p d) / F^{2}(d d)$ as a function of $\sigma_{p} / \sigma_{d}$ for the principal quantum number 3 of the Slater d-orbitals nicely approximates as $\left(\sigma_{p} / \sigma_{d}\right)^{2}$ with the Hilbert square norm of the difference between the exact ratio and the quadratic estimate being less than $2 \times 10^{-3}$ for the integration interval $[0,1]$ and becoming less than $2 \times 10^{-4}$ for a more realistic interval $[0.2,0.8]$ of the $\sigma_{p} / \sigma_{d}$ values.
} 


$$
\begin{aligned}
& V_{m m^{\prime}}^{L}=5 \sqrt{4 \pi} \sum_{k=0,2,4} \frac{(-1)^{m}}{\sqrt{2 k+1}}\left(\begin{array}{ccc}
2 & k & 2 \\
0 & 0 & 0
\end{array}\right) \\
& \quad \times\left(\begin{array}{ccc}
2 & k & 2 \\
m & m^{\prime}-m & -m^{\prime}
\end{array}\right) F_{k}\left(R_{L}\right) Y_{k}^{m-m^{\prime}}\left(\theta_{L}, \phi_{L}\right)
\end{aligned}
$$

where $\left(R_{L}, \theta_{L}, \phi_{L}\right)$ are the spherical coordinates of the ligand atom $L$ (the TMI is located in the center of the coordinate frame); $Y_{k}^{m-m^{\prime}}\left(\theta_{L}, \phi_{L}\right)$ are the spherical functions with the phases defined following Condon and Shortley [20]. Functions $F_{k}\left(R_{L}\right)$ are the integrals of squares of the radial parts $R_{n l}(r)$ of the atomic $d$-functions:

$$
\begin{aligned}
F_{k}(R)=R^{-(k+1)} \int_{0}^{R} r^{k} R_{n l}^{2}(r) r^{2} d r & \\
& \quad+R^{k} \int_{R}^{\infty} r^{-(k+1)} R_{n l}^{2}(r) r^{2} d r
\end{aligned}
$$

and depend on the distance $R_{L}$ from the atom of metal to the atom $L$. If the Slater AOs are taken for $R_{n l}(r)$, the $F_{k}(R)$ functions have been evaluated and are given, e.g., in Ref. [6].

The approximation in Eq. (54) is to assume all two-center matrix elements of the form

$$
\left(m m^{\prime} \mid l l^{\prime}\right)
$$

to be diagonal with respect to AOs $l l^{\prime}$ and to be equal for all AOs centered on the same atom $L$ of the ligand. Despite this, the components of Eq. (54) with $k=2,4$ describe precisely the interaction between the fluctuations in the $d$-shell and the electronic density in the $l$-system. The average of this operator produces the ionic contribution $W_{\mu \nu}^{\text {ion }} \mathrm{Eq}$. (25) to the effective crystal field as in the classical CFT. The fluctuations occurring on top of this average are responsible for the effect interesting to us. Generalizing the moves of Section 3.2.1., we obtain the correction to the matrix element $\left(d d^{\prime} \mid d^{\prime \prime} d^{\prime \prime \prime}\right)$ in the form of Eq. (38) and then inserting explicit forms of the matrix elements Eq. (55) we recast it in the form of renormalization of the Slater-Condon parameters $F_{2}$ and $F_{4}$. After substituting the matrix elements and of the elements of the polarization propagator supermatrix of the form $\Pi_{l l^{\prime} l^{\prime}}$ as stipulated by the approximation Eq. (54) to Eq. (38) we immediately get:

$$
\begin{aligned}
& \delta\left(m m^{\prime} \mid m^{\prime \prime} m^{\prime \prime \prime}\right)
\end{aligned}
$$

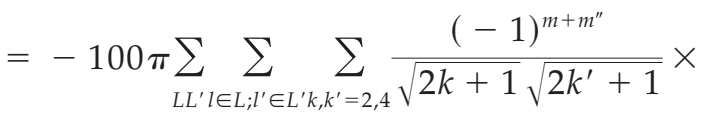

$$
\begin{aligned}
& \times\left(\begin{array}{ccc}
2 & k & 2 \\
0 & 0 & 0
\end{array}\right)\left(\begin{array}{ccc}
2 & k^{\prime} & 2 \\
0 & 0 & 0
\end{array}\right) \times \\
& \times\left(\begin{array}{cccc}
2 & k & 2 & \\
m & m^{\prime} & -m & -m^{\prime}
\end{array}\right) \\
& \times\left(\begin{array}{cccc}
2 & k^{\prime} & 2 & \\
m^{\prime \prime} & m^{\prime \prime \prime} & -m^{\prime \prime} & -m^{\prime \prime \prime}
\end{array}\right) \times \\
& \times F_{k}\left(R_{L}\right) Y_{k}^{m-m^{\prime}}\left(\theta_{L}, \phi_{L}\right) \\
& F_{k^{\prime}}\left(R_{L^{\prime}}\right) Y_{k^{\prime}}^{m^{\prime \prime}-m^{\prime \prime \prime}}\left(\theta_{L^{\prime}}, \phi_{L^{\prime}}\right) \sum_{\substack{i \in \text { occ } \\
j \in \text { vac }}} \frac{C_{i l} C_{j l} C_{i l^{\prime}} C_{j l^{\prime}}}{\varepsilon_{j}-\varepsilon_{i}} .
\end{aligned}
$$

Summation over the one-electronic states $l \epsilon L ; l^{\prime}$ $\epsilon L^{\prime}$ centered on ligand atoms $L L^{\prime}$ can be performed and straightforwardly yields:

$$
\begin{aligned}
& \delta\left(m m^{\prime} \mid m^{\prime \prime} m^{\prime \prime \prime}\right)=-100 \pi \sum_{L L^{\prime} k, k^{\prime}=2,4} \frac{(-1)^{m+m^{\prime \prime}}}{\sqrt{2 k+1} \sqrt{2 k^{\prime}+1}} \\
& \times \times\left(\begin{array}{ccc}
2 & k & 2 \\
0 & 0 & 0
\end{array}\right)\left(\begin{array}{ccc}
2 & k^{\prime} & 2 \\
0 & 0 & 0
\end{array}\right) \times \\
& \times\left(\begin{array}{ccc}
2 & k & 2 \\
m & m^{\prime}-m-m^{\prime}
\end{array}\right)\left(\begin{array}{ccc}
2 & k^{\prime} & 2 \\
m^{\prime \prime} & m^{\prime \prime \prime}-m^{\prime \prime}-m^{\prime \prime \prime}
\end{array}\right) \times \\
& \quad \times F_{k}\left(R_{L}\right) Y_{k}^{m-m^{\prime}}\left(\theta_{L}, \phi_{L}\right) F_{k^{\prime}}\left(R_{L^{\prime}}\right) Y_{k^{\prime}}^{m^{\prime \prime}-m^{\prime \prime}}\left(\theta_{L^{\prime}}, \phi_{L^{\prime}}\right) \pi_{L L^{\prime}}
\end{aligned}
$$

giving the result in terms of atom-atomic polarizabilities $\pi_{L L^{\prime}}$ of the $l$-system as coming from the semiempirical SCF calculation of the latter assumed by the EHCF theory. This expression is of course not spherically symmetric any more. By itself it does not represent any problem since in the TMC there is no such symmetry and in this respect the result is correct. However, both the general practice of spectroscopy of TMCs and the EHCF theory base the description of electron-electron interactions in the $d$-shell on a tacit assumption of spherical symmetry of these latter (usage of the $B$ and $C$ Racah parameters or the Slater-Condon parameters). For this reason, we consider below a symmetric version of the atomically resolved version of the polarization stipulated nephelauxetic effect by singling out the spherically symmetric part of the renormalization and reducing it to that of the Slater-Condon parameters $F_{2}(d d)$ and $F_{4}(d d)$. 
For this end, we assume the environment of the TMI in the TMC (the $l$-system) to be approximately spherically symmetric so that its excitation spectrum can be classified according to irreducible representations of the $\mathrm{SO}(3)$ group, i.e., as having definite angular momentum and its $z$-projection. Then the polarization propagator $\Pi$ is diagonal in the basis of such states:

$$
\begin{array}{r}
\Pi\left(\mathbf{R}, \mathbf{R}^{\prime}\right)=\sum_{k, \kappa} \Pi^{(k \kappa)}\left(\mathbf{R}, \mathbf{R}^{\prime}\right)=-\sum_{k, \kappa} \frac{|k \kappa\rangle\langle k \kappa|}{\omega_{k}} \Pi^{(k \kappa)}\left(\mathbf{R}, \mathbf{R}^{\prime}\right) \\
=-\frac{f_{k}^{*}(R) f_{k}\left(R^{\prime}\right)}{\omega_{k}} Y_{k}^{\kappa *}\left(\frac{\mathbf{R}}{R}\right) Y_{k}^{k}\left(\frac{\mathbf{R}^{\prime}}{R^{\prime}}\right)
\end{array}
$$

which can be understood as a sum over excitations of a spherical layer having the excitation spectrum of energies $\left\{\omega_{k}\right\}$. The ground state of the layer is spherically symmetric $(k=0)$. The spatial angular dependence of the state $k \kappa>$ is that of the spherical harmonic $Y_{k}^{k}(\theta, \phi)$, which is complemented by some radial dependence $f_{k}(R)$ of the one-electron transition density to obtain its total spatial dependence. This yields the expression for the polarization propagator of the effective medium representing the $l$-system in the coordinate representation. Using the $k \kappa$-resolved polarization propagator leads to the selection rules

$$
\begin{gathered}
k=k^{\prime} \\
m-m^{\prime}=\kappa=-\left(m^{\prime \prime \prime}-m^{\prime \prime}\right)
\end{gathered}
$$

(one can think that one of the operators $\hat{V}_{m m^{\prime}} \mathrm{mm}^{\prime}$ ignites in the $l$-system an excitation with definite $k \kappa$, which must be quenched by the $\hat{V}_{m^{\prime \prime} m^{\prime \prime}} m^{\prime \prime} m^{\prime \prime \prime}$ one), which reduce the general expression Eqs. (58) and (59) to

$$
\begin{aligned}
& \delta\left(m m^{\prime} \mid m^{\prime \prime} m^{\prime \prime \prime}\right) \\
& =-100 \pi \sum_{L L^{\prime} k=2,4} \frac{(-1)^{m+m^{\prime \prime}}}{2 k+1}\left(\begin{array}{lll}
2 & k & 2 \\
0 & 0 & 0
\end{array}\right)^{2} \times \\
& \times\left(\begin{array}{cccc}
2 & k & 2 \\
m & m^{\prime}-m & -m^{\prime}
\end{array}\right)\left(\begin{array}{ccc}
2 & k & 2 \\
m^{\prime \prime} & m^{\prime \prime \prime}-m^{\prime \prime} & -m^{\prime \prime \prime}
\end{array}\right) \\
& \times \\
& \times \sum_{\kappa} \Pi^{(k \kappa)}\left(\mathbf{R}_{L^{\prime}}, \mathbf{R}_{L^{\prime}}\right) \\
& \times F_{k}\left(R_{L}\right) Y_{k}^{\kappa}\left(\theta_{L^{\prime}} \boldsymbol{\phi}_{L}\right) F_{k}\left(R_{L^{\prime}}\right) Y_{k}^{-\kappa}\left(\theta_{L^{\prime}}, \phi_{L^{\prime}}\right) .
\end{aligned}
$$

It is easy to check that the phase factor together with the 3jm symbols yields precisely the coefficient of the Slater-Condon parameter $F_{k}(d d)$ in the expansion of the two-electron matrix element $\left(m m^{\prime} \mid m^{\prime \prime} m^{\prime \prime \prime}\right)$ so that the given result can be represented as a renormalization of the Slater-Condon parameters, namely:

$$
\begin{aligned}
& \delta F_{k}(d d)=-\frac{4 \pi}{(2 k+1)} \sum_{L L^{\prime}} \sum_{\kappa} \Pi^{(k \kappa)} \\
& \left(\mathbf{R}_{L}, \mathbf{R}_{L^{\prime}}\right) F_{k}\left(R_{L}\right) Y_{k}^{\kappa}\left(\theta_{L}, \phi_{L}\right) F_{k}\left(R_{L^{\prime}}\right) Y_{k}^{-\kappa}\left(\theta_{L^{\prime}}, \phi_{L^{\prime}}\right) .
\end{aligned}
$$

On the other hand, the atom-atomic polarizabilities $\pi_{L L^{\prime}}$ of the $l$-system entering Eq. (59) correspond to the values of $\Pi\left(R, R^{\prime}\right)$ through the standard relation between the coordinate and the $\mathrm{AO}$ representations. Indeed, for a system described in the AO representation, the orbital-orbital mutual polarizability $\Pi_{l l l^{\prime} l^{\prime}}$

$$
\Pi_{l l l^{\prime} l^{\prime}}=\sum_{\substack{i \varepsilon \text { occ } \\ j \varepsilon v a c}} \frac{C_{i l} C_{j l} C_{i l l^{\prime}} C_{j l^{\prime}}}{\varepsilon_{j}-\varepsilon_{i}}
$$

contributes to that in the coordinate representation according to:

$$
\Pi\left(\mathbf{R}, \mathbf{R}^{\prime}\right)=\sum_{L L^{\prime} l \varepsilon L ; j^{\prime} \varepsilon L^{\prime}}\left|\varphi_{l}(\mathbf{R})\right|^{2}\left|\varphi_{l^{\prime}}\left(\mathbf{R}^{\prime}\right)\right|^{2} \Pi_{l l l^{\prime} l^{\prime}}
$$

where $\varphi l(\mathbf{R})$ and $\varphi_{l^{\prime}}\left(\mathbf{R}^{\prime}\right)$ are the explicit forms of the $l$-th and $l^{\prime}$-th AOs giving necessary spatial density distributions. The approximation of Eq. (55) is equivalent to setting

$$
\left|\varphi_{l}(\mathbf{R})\right|^{2}=\delta^{(3)}\left(\mathbf{R}-\mathbf{R}_{L}\right)
$$

for all $l \epsilon L$. This immediately results in

$$
\Pi\left(\mathbf{R}, \mathbf{R}^{\prime}\right)=\sum_{L L^{\prime}} \delta^{(3)}\left(\mathbf{R}-\mathbf{R}_{L}\right) \delta^{(3)}\left(\mathbf{R}^{\prime}-\mathbf{R}_{L^{\prime}}\right) \pi_{L L^{\prime}}
$$

This move allows to single out the spherically symmetric part of the renormalization:

$$
\begin{aligned}
& \delta F_{k}(d d)= \\
& -\frac{4 \pi}{(2 k+1)} \sum_{L L^{\prime}} F_{k}\left(R_{L}\right) Y_{k}^{\kappa}\left(\theta_{L^{\prime}}, \phi_{L}\right) F_{k}\left(R_{L^{\prime}}\right) Y_{k}^{-\kappa}\left(\theta_{L^{\prime}}, \phi_{L^{\prime}}\right) \pi_{L L^{\prime}}
\end{aligned}
$$

which can be as well understood as a double-generalized Fourier transform of the point-point polar- 
ization propagator calculated in a finite number of points referring to the positions of the ligand atoms.

The generalized Fourier transform can be used to establish the relation with the continuous insulator model result Eq. (27). For that end, we represent in Eq. (62) the sum over $L L^{\prime}$ as an integral over $\mathbf{R}, \mathbf{R}^{\prime}$ of an expression containing the $L L^{\prime}$ sum of the $\delta^{(3)}$ densities centered in $R_{L}, R_{L^{\prime}}$ as a multiplier. Going to the spherical coordinates for the both integration variables $\mathbf{R}, \mathbf{R}^{\prime}$ and performing integration over the angular variables, we arrive to the following expression for the polarization-driven interaction renormalization:

$$
\delta F_{k}(d d)=-\frac{4 \pi}{(2 k+1) \omega_{k}}\left[\int_{b}^{\infty} d R R^{2} f_{k}(R) F_{k}(R)\right]^{2} .
$$

According to Ref. [6], $F_{k}(R) \sim 1 / R^{k+1}$, then as-

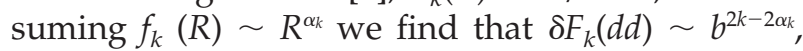
which agrees with Eq. (27) provided for all $k \alpha_{k}=$ $-1 / 2$. This is precisely the decay exponent for the radial function of the wave in the spherical layer as given by a Bessel function with a half-integer index, which one can expect to be a model for the $l$-system.

\section{Outlook and Discussion}

The derivation of the formulae for describing nephelauxetic effect in TMCs in terms of the polarization propagator and reducing atom-atom polarizability for those forming the $l$-system of the TMCs naturally poses the question on the sources of these quantities (polarizabilities). General expressions for it like used in Eq. (58) should not deceive the reader: in combination with semiempirical methods using the valence basis only some (indefinite) part of the total polarizability propagator can be reproduced. In the case of organic molecules where the number of occupied and empty orbitals is somewhat balanced it may be not that critical and acceptable estimates of polarizabilities can be obtained in semiempirical context (see Ref. [21]). The situation comes to a visible disaster with the strongest renormalizers: late halogene anions $\left(\mathrm{Br}^{-}, \mathrm{I}^{-}\right)$or halcogene dianions $\left(\mathrm{Se}^{2-}, \mathrm{Te}^{2-}\right)$. When treated semiempirically these important ligands cannot have any polarizabilty at all-no vacant orbitals are present. In this situation, one has to accept extending the set of necessary semiempirical parameters on account of polarizabilities. It should not be con- sidered as a catastrophe: so-called ab initio methods require much more parameters to describe their respective basis sets or pseudopotentials used, none of which individually has any physical sense. Polarizabilities, however, have transparent physical meaning and can also be used in a line with the proposition of developing a "new generation" of semiempirical methods [22] characterized among other features by extending these latter by including dispersion forces ultimately determined by the same polarizabilities and being in a way another face of the same effect: of interactions between the fluctuations of electron densities in different parts of the system as omitted from the Hartree-Fock or other mean field picture. An alternative might be developing estimates of the polarizabilities of the closed shell anions with no vacant orbitals on the basis of some analysis of interplay between the valence shell electrons considered explicitly and both the core and empty states not included in the calculation; however, this option will be postponed for the future.

\section{ACKNOWLEDGMENTS}

This article is dedicated to Professor István Mayer on the occasion of his 65th birthday. While knowing that not numbers, but physical content concerns him most of all, the authors tried to make it to be interesting for him to read.

\section{References}

1. (a) Jaurgensen, C. K. Prog Inorg Chem 1964, 4, 73; (b) Jaurgensen, C. K. Adv Chem Phys 1963, 5, 33.

2. Bethe, H. A. Ann Phys 1929, 3, 133.

3. Ballhausen, C. J. Introduction to Ligand Field Theory; McGraw Hill: New York, 1962.

4. (a) Jaurgensen, C. K. Absorption Spectra and Chemical Bonding in Complexes; Pergamon Press: Oxford, 1962; (b) Jaurgensen, C. K. Modern Aspects of Ligand Field Theory; North-Holland: Amsterdam, 1971.

5. Lever, A. B. P. Inorganic Electronic Spectroscopy; Elsevier: Amsterdam, 1984.

6. Bersuker, I. B. Electronic Structure and Properties of Coordination Compounds; Khimiya: Moscow, 1976 [in Russian].

7. Soudackov, A. V.; Tchougréeff, A. L.; Misurkin, I. A. Theor Chim Acta 1992, 83, 389.

8. McWeeny, R. Methods of Molecular Quantum Mechanics, 2nd ed.; Academic Press: London, 1992.

9. Löwdin, P.-O. Perturbation Theory and Its Application in Quantum Mechanics; Wiley: New York, 1966. 
10. Soudackov, A. V.; Tchougréeff, A. L.; Misurkin, I. A. Int J Quantum Chem 1996, 58, 161.

11. Soudackov, A. V.; Tchougréeff, A. L.; Misurkin, I. A. Russ J Phys Chem 1994, 68, 1256.

12. Soudackov, A. V.; Tchougréeff, A. L.; Misurkin, I. A. Russ J Phys Chem 1994, 68, 1264.

13. Tokmachev, A. M.; Tchougréeff, A. L. Chem Phys Rep 1999, 18,80 .

14. Morrison, C.; Mason, D. R.; Kikuchi, C. Phys Lett A 1967, 24, 607.

15. Tchougréeff, A. L. Phys Chem Chem Phys 1999, 1, 1051.

16. Tchougréeff, A. L. J Struct Chem 2007, 48, S39 [in Russian].
17. Tchougréeff, A. L. Hybrid Methods of Molecular Modeling; Springer Verlag: London, 2008.

18. Hammermesh, M. Group Theory and Its Applications to Physical Problems; Addison Wesley, Reading: Massachusetts, 1964.

19. Tchougréeff, A. L. Int J Quantum Chem 2007, 107, 2519.

20. Condon, E. U.; Shortley, G. H. Theory of Atomic Spectra: Cambridge University Press: Cambridge, 1951.

21. Tchougréeff, A. L. DSc Thesis, Karpov Institute, Moscow, Russia, 2004 [in Russian].

22. Winget, P.; Selçcuki, C.; Horn, A. H. C.; Martin, B.; Clark, T. Theor Chem Acc 2003, 110, 254. 